\title{
Influence of the Gender on Cerebral Vascular Diameters Observed during the Magnetic Resonance Angiographic Examination of Willis Circle
}

\author{
Marco Antonio Stefani*, Felipe Luis Schneider, Antonio Carlos Huf Marrone and Antonio \\ Generoso Severino \\ Departamento de Ciência Morfologica; Universidade Federal do Rio Grande do Sul; Rua Sarmento Leite 500, \\ 90050-170; Porto Alegre - RS - Brasil
}

\begin{abstract}
The present study evaluated the calibers and anatomic configurations based on the Magnetic Resonance analysis (MRA), assaying the cerebral vascular territories and sex-linked variations. A randomized sample of 30 angiographic examinations in adult patients of both sexes was obtained and components of the circle of Willis were identified. Branch diameters were measured on a transversal cut $5 \mathrm{~mm}$ from the vessel origin in a typical angiographic frontal incidence. For the comparative statistical analysis, tests were divided in the groups considering the patients' sex and age. The classical Circle of Willis configuration was oberved in only 15 samples $(50 \%)$. Greater calibers were observed in the arteries of the posterior circulation and multiple linear regression analysis established that the caliber of the posterior circulation was influenced by an independent variable related to the gender. Additional variations included unilateral and bilateral fetal and hypoplasic Posterior communicating arteries. In the anterior cerebral artery (ACA), the presence of an accessory developed ACA, an ACA giving branches to the distal portion of the two hemispheres and a third median ACA the variants were observed. Gender influenced the variations on internal diameters of posterior circulation vessels, with larger measurements in men.
\end{abstract}

Key words: angiography, magnetic resonance, anatomic variations

\section{INTRODUCTION}

The arise of new non-invasive human body investigation techniques has enabled the identification and the study of cerebral circulation in living individuals. Nuclear magnetic resonance (MR), in particular, has contributed to this knowledge, where upon the assessment of these examinations have revealed data beyond the classical findings of anatomy, such as vascular patterns present in different genders and ages. The present study analyzed the calibers and anatomic variations based on the MR results of individuals with no vascular disease, while comparing various vascular territories and anatomic variations.

\section{MATERIALS AND METHODS}

A random sampling of 30 magnetic resonance angiography examinations from both male and female adults was made. In order to perform the tests, a $1.5 \mathrm{t}$ MR equipment and the images were processed by a 3D Advantage Windows software ${ }^{\circledR}$ (GE Medical Systems) in a SUN ULTRA $1{ }^{\circledR}$ workstation. Although the tests were chosen arbitrarily from the MR Service database, only

*Author for correspondence: marco.stefani@ufrgs.br 
those with normal radiological reports and appropriate technical standards were included in the study. The following branches and components of the Willis polygon were identified: internal carotid artery (ICA), anterior cerebral artery (ACA), posterior cerebral artery (PCA), basilar artery, anterior communicant artery (ACommA), middle cerebral artery (MCA), and posterior communicant artery (PCommA).

The ACA (proximal A1 segment), MCA (proximal M1 segment), PCA (P1 segment, precommunicating segment), and the basilar artery diameters were measured through a transversal cut placed $5 \mathrm{~mm}$ from the vessel origin in a typical angiographic frontal incidence. This was chosen to standardize the obtained measurements and allow further study reproduction. In order to better visualize the posterior communicant artery
(PCommA), thin axial cuts were made $(1 \mathrm{~mm})$ in the posterior region of the carotid siphon (Fig. 1).

For comparative statistical analysis purposes, the tests were divided into groups while taking into account patients' sex and age. Two experts blinded and performed the assessment of vascular distributions and calibers, and a multivariate analysis was performed to study different variables that could interfere with the results.

\section{RESULTS}

The group consisted of an equal number of men and women, with a mean age of $45+/-3$ years. Age differences related to the sex were not taken into account. Branch calibers arising from the circle of Willis (CW) are shown in Table 1.

Table 1 - The transversal diameters of the branches part of the circle of Willis (in millimeters).

\begin{tabular}{lcccc}
\hline Vessel & Mean & Standard Error & Max & Min \\
\hline ACA R & 2.0 & 0.07 & 2.9 & 1.1 \\
ACA L & 2.2 & 0.09 & 3.3 & 1.5 \\
Total ACA & 2.1 & 0.06 & 3.3 & 1.1 \\
MCA R & 2.7 & 0.07 & 3.6 & 1.9 \\
MAC L & 2.7 & 0.06 & 3.6 & 1.9 \\
Total MCA & 2.7 & 0.05 & 3.6 & 1.9 \\
BA & 2.9 & 0.09 & 3.7 & 2.1 \\
PCA L & 2.1 & 0.07 & 3.0 & 1.2 \\
PCA R & 2.0 & 0.08 & 2.8 & 1.0 \\
Total PCA & 2.1 & 0.06 & 3.0 & 1.0 \\
\hline
\end{tabular}

The analyzed calibers presented a normal distribution. There were no notable differences among the MCA, PCA, and ACA branches in regards to the side of the hemisphere ( $p>0.05$ ). During the reconstruction of two fetal pattern cases with $\mathrm{CW}$ distribution, the segments were not visualized. However, it was still possible to outline them as individualized vessels on thin axial cuts $(1 \mathrm{~mm})$ in the posterior region of the carotid siphon (Fig. 1).
As shown on Table 2, there were larger calibers in the male arteries of the posterior circulation (Basilar artery and PCA), with a $\mathrm{p}<0.001$. Another analysis indicated that the branches of the posterior circulation also presented larger diameters in the individuals older than 40 years $(p<0.05)$. However, a multiple linear regression study established that the caliber of the posterior circulation was influenced by an independent variable related to the gender $(\mathrm{p}=0.05)$. 


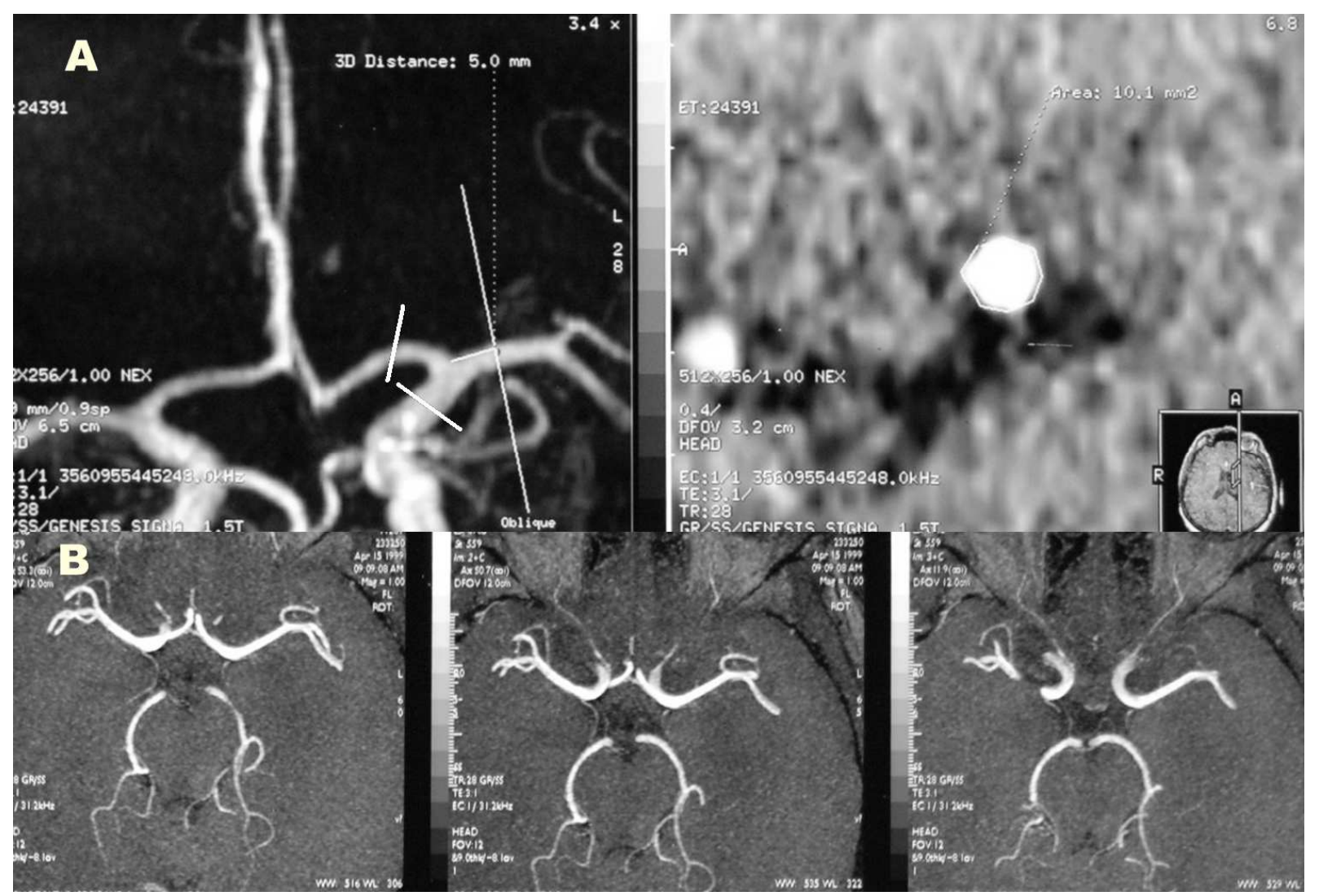

Figure 1 - (A) Standartizad method for vessel diameter measurement, with transversal cuts $5 \mathrm{~mm}$ from the vessel origin in a typical angiographic frontal incidence. (B)Axial cuts of $1 \mathrm{~mm}$ in the posterior region of the carotid siphon to better visualize the posterior communicating artery.

Table 2 - Vessel diameters according with subgroups.

\begin{tabular}{ccccccccc}
\hline Vessel & $\begin{array}{c}\mathbf{F}(\mathbf{n}=15) \\
\text { Mean }\end{array}$ & $\begin{array}{c}\text { Standard } \\
\text { Error }\end{array}$ & $\begin{array}{c}\text { M(n=15) } \\
\text { Mean }\end{array}$ & $\begin{array}{c}\text { Standard } \\
\text { Error }\end{array}$ & $\begin{array}{c}>\text { 40y/o(n=16) } \\
\text { Mean }\end{array}$ & $\begin{array}{c}\text { Standard } \\
\text { Error }\end{array}$ & $\begin{array}{c}\text { Mey/o(n=14) } \\
\text { Mean }\end{array}$ & $\begin{array}{c}\text { Standard } \\
\text { Error }\end{array}$ \\
\hline ACA & 2 & 0,32 & 2,2 & 0,52 & 2,1 & 0,43 & 2,1 & 0,45 \\
MCA & 2,6 & 0,4 & 2,7 & 0,34 & 2,7 & 0,36 & 2,6 & 0,38 \\
PCA & 1,9 & 0,44 & 2,3 & 0,37 & 2,2 & 0,42 & 1,9 & 0,46 \\
BA & 2,5 & 0,37 & 3,2 & 0,41 & 3 & 0,45 & 2,7 & 0,53 \\
Mean age & 42 & 16,6 & 47 & 16,6 & 57 & 12 & 31 & 7,55 \\
\hline
\end{tabular}

$\overline{\mathrm{ACA}}=$ anterior cerebral artery; $\mathrm{MCA}=$ middle cerebral artery ; $\mathrm{PCA}=$ posterior cerebral artery $; \mathrm{BA}=$ basilar artery $; \mathrm{M}=\mathrm{male}$; $\mathrm{F}=$ female; $y / o=y e a r s$ old.

Five vascular patterns of the Willis polygon configuration were found, as demonstrated in Figure 2. The classical vessel distribution standard in the polygon was most consistent, as observed in 15 tests $(50 \%)$. In this group, the anterior communicating artery was more evident as an isolated vessel in seven cases. Additionally, there was asymmetry of the ACA segments in 15 cases and hypoplasia was observed in two by using the criteria suggested by (Zurada and Gielecki 2007).
Other variations included the hypoplasia of one (four cases) or both PCommA (two cases) and the presence of unilateral (one case) and bilateral (three cases) fetal PCommA associated with a clear hypoplasia of the proximal segment of the PCA. The distribution, commonly found in the anterior cerebral artery, indicated the presence of two ACAs running parallel alongside the genu of the corpus callosum (20 cases), each giving branches to the ipsilateral hemisphere (classical type). 


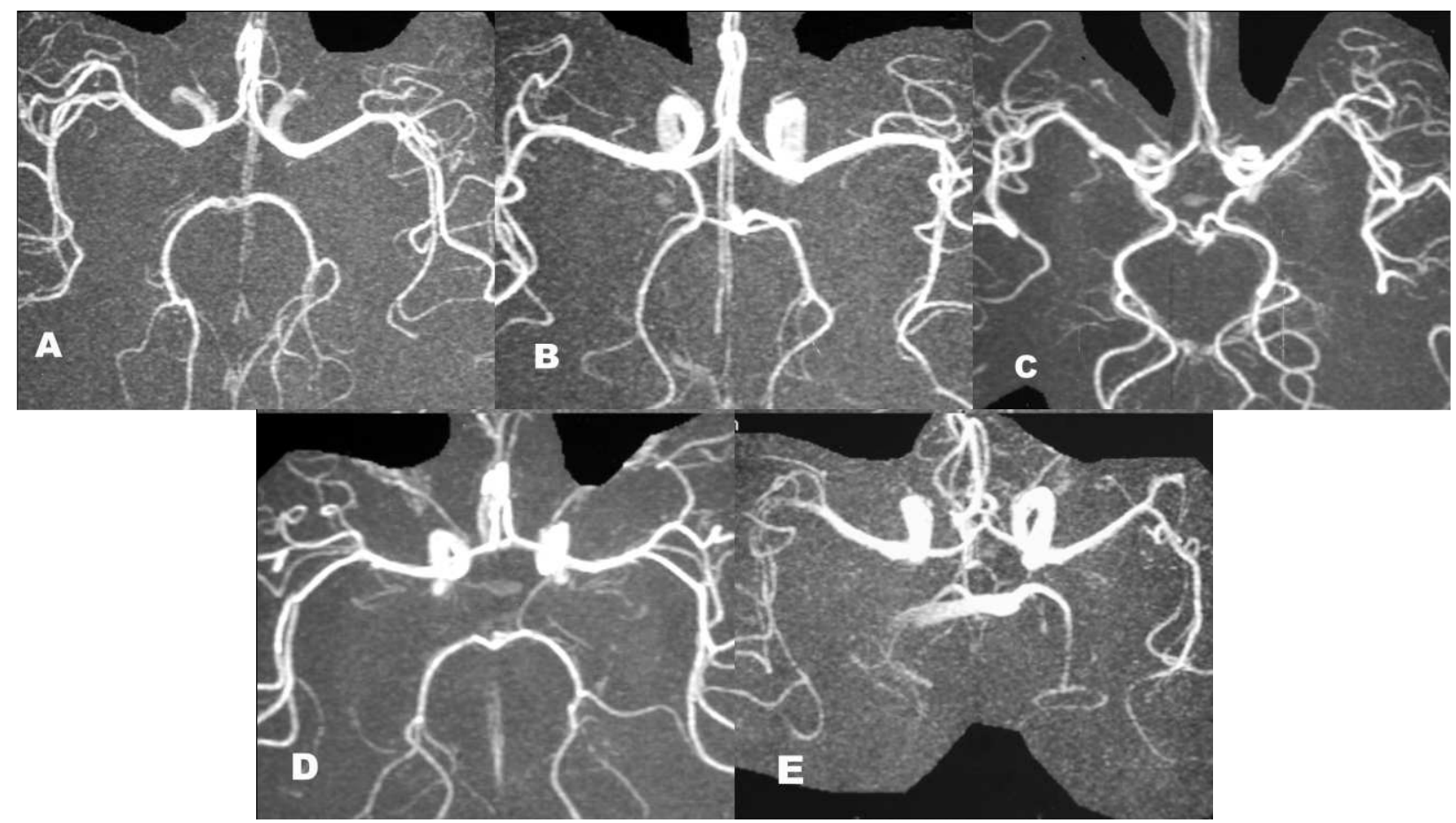

Figure 2 - Vascular patterns of Willis polygon configuration in a superior angiographic view: (A) classical standard of distribution, (B) presence of unilateral and (C) bilateralfetal PCommA, (D) hypoplasia of one and (E) both PCommA.

The anatomical distribution study of the distal ACA branches was very complex and findings were not consistent in all the cases. However, there were some variations of the ACA branching patterns subsequent to the discovery of the anterior communicating artery, such as the presence of an accessory-developed ACA (two cases), an ACA giving branches to the distal portion of the two hemispheres (two cases) and a third median ACA, irrigating the two hemispheres in one case (Fig. 3). In nine cases, the callosomarginalis artery was observed in only one hemisphere, and in three cases, it was present in both the hemispheres. In all of these situations, the distal cortical ACA branches could not be individualized clearly, thus affecting the study of these vessels.

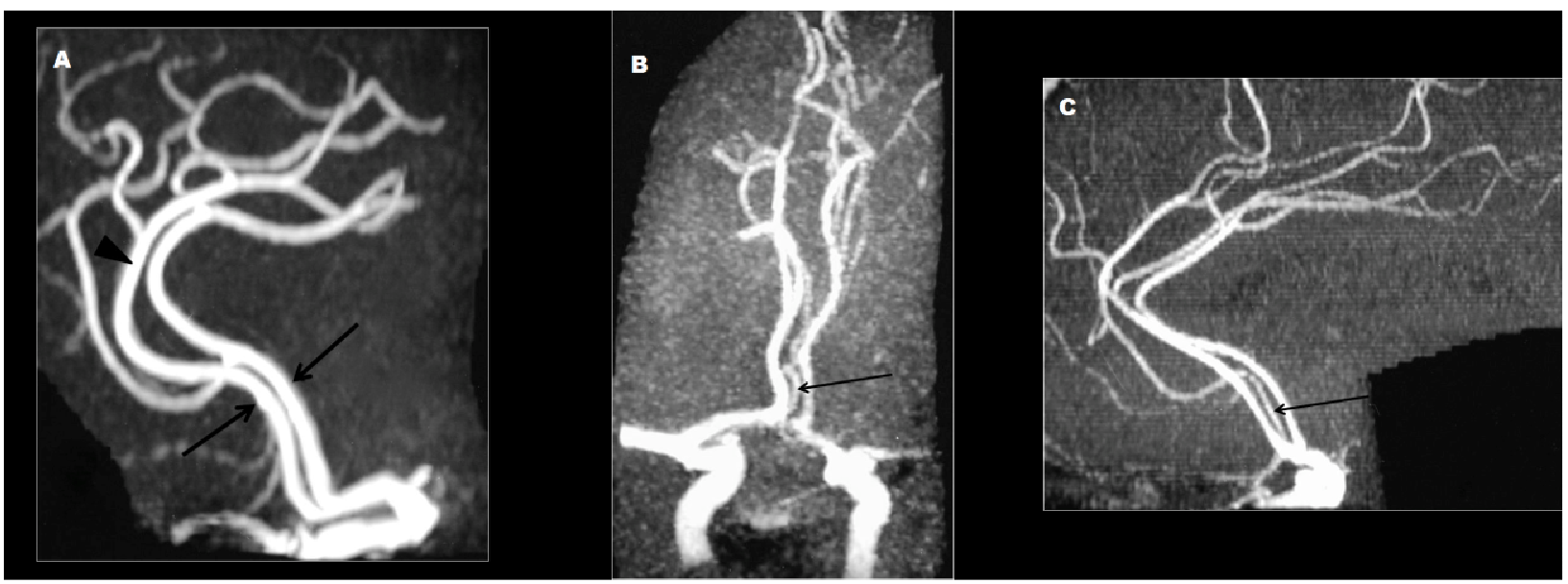

Figure 3 - (A)Lateral angiographic view with two ACAs running parallel around of the genu of the corpus callosum (arrows), with the emergence of the callosomarginal artery from one (arrow head);(B) accessory developed ACA (arrow); (C)lateral view showing a third median ACA irrigating the two hemispheres (arrow). 
The more frequent ramification pattern of the middle cerebral artery was the bifurcation (16 on the right, and 15 on the left), followed by the trifurcation (seven on the right, and three on the left), and the presence of a single MCA, without terminal branches (five on the right, and three on the left). In one case, four branches were observed arising from the MCA (Fig. 4).

The most common configuration found in the vertebrobasilar system was the one in which the PCA originated from the basilar artery in a symmetric form (classical type - 18 cases). As aforementioned, proximal PCA hypoplasia was unilateral in one case and bilateral in three (Fig. 5). The superior cerebellar artery had a variety of configurations, with the classical anatomical pattern present in $70 \%$ of the (21/30) cases (Fig.5). There were unilateral duplicate vessels in five cases and bilateral vessels in one. In three cases, one of the superior cerebellar arteries (SCA) originated from the proximal PCA.

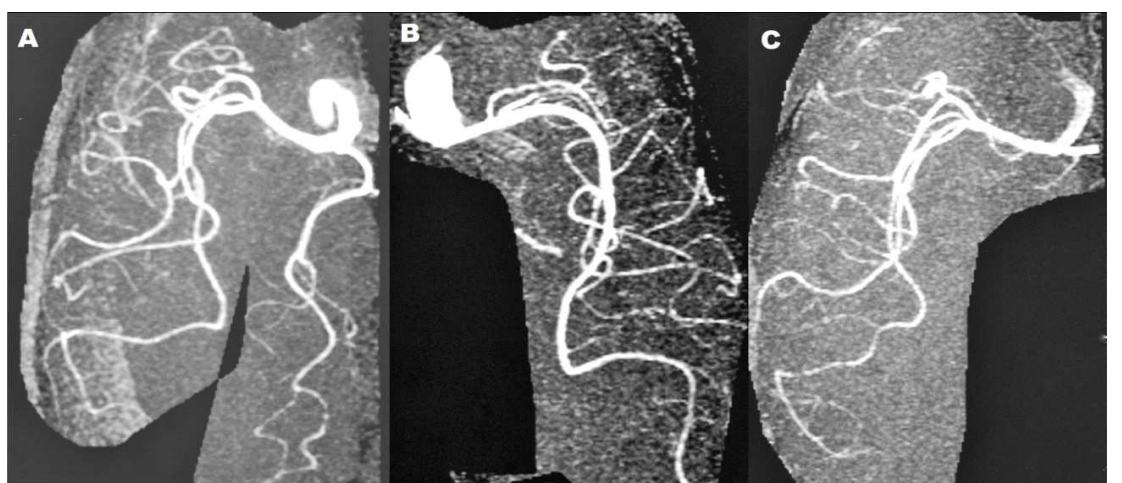

Figure 4 - (A) Superior view of middle cerebral artery as it ends as a trifurcation, (B) a single MCA branch and the presence of (C) four terminal branches.

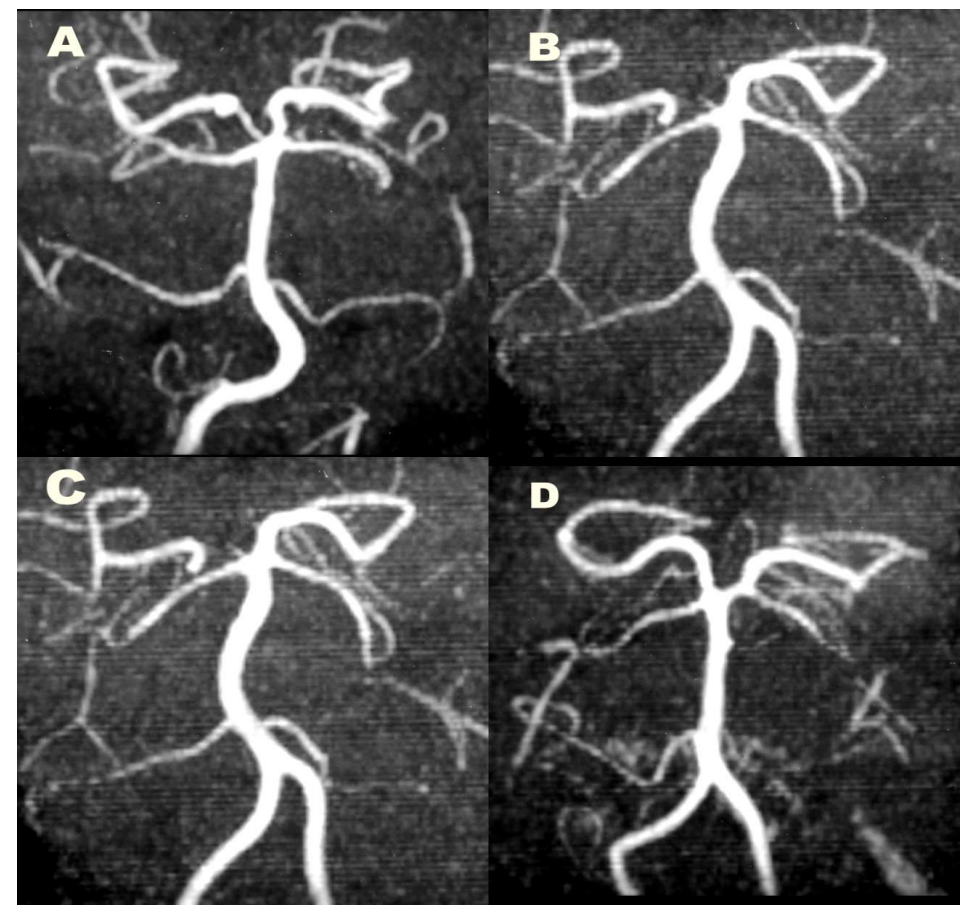

Figure 5 - Frontal angiographic view showing (A) bilateral and (B) unilateral fetal PCommA, with proximal PCA hypoplasia (arrows). The same view of a case with (C) bilateral duplicate superior cerebellar vessels and (D) the origin of this vessel directly from the proximal PCA (arrow). 


\section{DISCUSSION}

There are reports describing the presence of a complete Willis polygon in MR examinations in 34 to $42 \%$ of the cases (Milisavljevic et al. 1986; Macchi et al. 1996; krabbe-Hartkamp et al. 1998; $\mathrm{He}$ et al. 2007). The most common anatomical variations observed were the presence of a fetal posterior communicant artery and hypoplasia of the proximal segment of the ACA. These modifications did not seem to differ in different populations, although they could be more regularly associated with the presence of cerebral aneurysms(Horikoshi et al. 2002). Overall, MR angiography performs a reliable reproduction of the cerebral vessel calibers and of the Willis polygon branches. These data were comparable to the ones obtained through the computerized tomography angiography(Katz et al. 1995).

As aforementioned, the configuration of the circle of Willis influences the aspect of the initial segment of the PCA. Due to the high frequency of fetal posterior communicating arteries, measurements of the initial PCA segment diameters may be misinterpreted. The standardized measurement of the confluence of the PCommA provided the real diameters of the distal part of the PCA, and these values did not vary from the ones reported in literature. It would be also worth mentioning here about the differences of the diameter in regards to gender as also pointed out in previous study (Horikoshi et al. 2002).

The MCA branch traverses the entire extension of the lateral groove, distributing the collaterals that give supply to a large extension of the lateral face of each hemisphere. It runs parallel alongside the sphenoid bone wing, as the M1 segment; whereas in the lateral portion of the Sylvian fissure, it curves posterior and superiorly in order to reach the insula, as the M2 segment. This partition may originate a single trunk or up to four branches. The MCA patterns observed in the present study were similar to the ones reported in literature, as the bifurcation was present in approximately two-third of the cases, followed by the bifurcation in almost $30 \%$ of the vessels (Gibo et al. 1981; Umansky et al. 1984). Other variations may also occur, such as the presence of a single trunk of MCA without the erminal branches, the presence of an accessory MCA, the origin of a precocious branch, or a treelike ramification in more than four terminal branches in the M1-M2 junction (Gibo et al. 1981; Umansky et al. 1984; Umansky et al. 1988).
The classically observed calibers of the medium cerebral artery varied from 2.4 to $4.6 \mathrm{~mm}$ (reported means of 3.0 to $3.9 \mathrm{~mm}$ ) and were obtained based on the measurements of the external diameters of the vessels previously injected with the resin or latex (Gibo et al. 1981; Gomes et al. 1984). However, the vascular calibers observed in the MR angiography studies were slightly shorter (mean of $2.7 \mathrm{~mm}$ ) in relation to the ones observed in the anatomic studies (krabbeHartkamp et al. 1998). This minimum difference existed probably because the MR angiography measured the internal diameter of the vessel while excluding the arterial wall. Artifacts inherent in the preparation of postmortem vascular injection also should be taken into account, such as the loss of vascular muscular tone, the type of injected material, the behavior of the injected substance once solidified, and the amount of filling and pressure exerted during vascular repletion.

The ACA - terminal medial branch of the ICA originates at the medial region of the Sylvian fissure, lateral to the optic chiasm with several anatomic dispositions (Jackowski et al. 1999; Stefani et al. 2000). There are discussions whether the AC should be called pericallosal artery in distal segment to the ACommA (Lin et al. 1974; Perlmutter and Rothon 1978), or only after the emergency of the callosomarginal branch (CmA)(Snickers and Drake 1973).

In almost $80 \%$ of the cases, there were two postcommunicant branches of equal caliber, following the anterior communicating artery, with several described anatomic variations in the distal segment of the ACA (Ozaki et al. 1977; Perlmutter and Rothon 1978; Jackowski et al. 1999; Stefani et al. 2000). These variations have been well identified anatomically (Stefani et al. 2000), albeit not always well evidenced in the MR angiography due to the close parallel course of the two anterior in the midline.

Similar to the MCA, there was little difference observed between the data obtained from the ACA external diameters - following the ICA bifurcation in anatomic preparations - and those obtained from the MRA (krabbe-Hartkamp et al. 1998). Anatomic findings revealed slightly larger average diameters varying from 2.4 to $2.6 \mathrm{~mm}$ in this segment (Perlmutter and Rothon 1978; Gomes et al. 1986; Stefani et al. 2000), against a mean of 2.2 $\mathrm{mm}$ in the image findings.

Discussions regarding the definition of hypoplasia and vessel asymmetry are often based on the 
visual aspect during the operations or angiographic examinations. In order to classify these terms, a mathematical equation has been proposed by using the percentage relationship of the two branches as a coefficient (Zurada and Gielecki 2007). According to these authors, coefficients between 10 and $40 \%$ would be defined as asymmetry, whereas $>40 \%$ would be defined as vessel hypoplasia. For this particular location, other authors have proposed a diameter of 0.3 to $1.0 \mathrm{~mm}$ as a parameter for the hypoplasia (Perlmutter and Rothon 1978; Milenkovic 1981). This last definition seemed more helpful in terms of clinical and neurosurgical practice, as it was linked with clinically relevant circumstances such as the association with the presence of ACommA aneurysms. In the present study, we have observed coefficients greater than $40 \%$ in 2 cases; however, the smaller vessel diameter was between 1.5 and $2.0 \mathrm{~mm}$ in these instances. The smallest diameter found $(1.1 \mathrm{~mm}$, in one case) had a vessel coefficient of $27 \%$, as the contra-lateral vessel was also small. Based on these numbers, this equation appeared inappropriate for the clinical or radiological practices.

In the present study, the classical standard of the polygon conformation were commonly observed, thus confirming the anatomic findings described in the literature. The identification of the Willis polygon branches and their anatomic variations could be done through the MR angiography, but the same was not applicable with the cortical distal branches. Although the identification of some anatomic variations was possible through this technique, the juxtaposition and tortuosity found in the distal ACA prevented a detailed image of this vessel. Also, the presence of AC crossed branches (directed towards the contra-lateral hemisphere) could be seen in the MRA.

Finally, when comparing the present study to the findings of anatomic specimens in the literature, there was no difference between the internal diameter measures and the branches' standard variation of the polygon vessels, thus corroborating the reproducibility of the MRA measures. The frequency of the anatomic variation was also in accordance with the ones described in the literature that have used anatomic preparations. Additionally, gender influenced the variations on both the PCA and BA internal diameters, with larger measurements in the males. It was, therefore, concluded that the MR angiography could be a good alternative for the anatomic study of the cerebral vascularization in vivo. Further angiographic studies in the cases associated with the abnormalities might provide more clinical and relevant definition of the term hypoplasia.

\section{ACKNOWLEDGEMENTS}

The authors wish to thank $\mathrm{Mr}$ Alessandro Mazzola, Dr Flávio Aesse and Dr Claudio Pitta Pinheiro for their help on the acquisition of the images.

\section{REFERENCES}

Gibo H, Carver CC, Rhoton AL, Jr., Lenkey C and Mitchell RJ. Microsurgical anatomy of the middle cerebral artery. Journal of Neurosurgery. 1981; 54(2): 151-69.

Gomes F, Dujovny M, Umansky F, Ausman JI, Diaz FG, Ray WJ, et al. Microsurgical anatomy of the recurrent artery of Heubner. Journal of Neurosurgery. 1984; 60(1): 130-39.

Gomes FB, Dujovny M, Umansky F, Berman SK, Diaz FG, Ausman JI, et al. Microanatomy of the anterior cerebral artery. Surg Neurol. 1986; 26(2): 129-41.

He J, Liu H, Huang B and Chi C. Investigation of morphology and anatomic variations of circle of willis and measurement of diameter of cerebral arteries by 3D-TOF angiography. Sheng $W u$ Yi.Xue.Gong.Cheng Xue.Za Zhi. 2007; 24(1): 39-44.

Horikoshi T, Akiyama I, Yamagata Z, Sugita M and Nukui H. Magnetic resonance angiographic evidence of sex-linked variations in the circle of willis and the occurrence of cerebral aneurysms. J.Neurosurg. 2002; 96(4): 697-703.

Jackowski AP, Meneses MS, Ramina R, Marrone AC, Stefani MA, Aquini MG, et al. Perforating and leptomeningeal branches of the anterior communicating artery: an anatomical review. Crit Rev.Neurosurg. 1999; 9(5): 287-94.

Katz DA, Marks MP, Napel SA, Bracci PM and Roberts SL. Circle of Willis: evaluation with spiral CT angiography, MR angiography, and conventional angiography. Radiology. 1995; 195(2): 445-49.

Kabbe-Hartkamp MJ, van der GJ, de Leeuw FE, de Groot JC, Algra A, Hillen B, et al. Circle of Willis: morphologic variation on three-dimensional time-offlight MR angiograms. Radiology. 1998; 207(1): 10311.

Kabbe-Hartkamp MJ, van der Grond J, Leeuw F, Groot JC, Algra A, Hillen B, et al. Circle of Willis: Morphologic variation on three-dimensional time-offlight MR angiograms. Radiology. 1998; 207: 103-11. 
Lin J, Kircheff I, Newton TH and Potts DG (1974). Normal anterior cerebral artery complex. Radiology of the skull and brain. St. Louis, CV Mosby. 1: 1319410.

Macchi C, Catini C, Federico C, Gulisano M, Pacini P, Cecchi $\mathrm{F}$, et al. Magnetic resonance angiographic evaluation of circulus arteriosus cerebri (circle of Willis): a morphologic study in 100 human healthy subjects. Ital.J.Anat Embryol. 1996; 101(2): 115-23.

Milenkovic Z. Anastomosis between internal carotid artery and anterior cerebral artery with other anomalies of the circle of Willis in a fetal brain. $J$ Neurosurg. 1981; 55: 701-03.

Milisavljevic M, Marinkovic S and Djordjevic L. Anastomoses in the territory of the posterior cerebral arteries. Acta Anat. 1986; 127: 221-25.

Ozaki T, Handa H, Tomitoto $\mathrm{K}$ and Hazama $\mathrm{F}$. Anatomical variations of the arterial system of the base of the brain. Arch Jap Chir. 1977; 46: 3-17.

Perlmutter D and Rothon AL, Jr. Microsurgical anatomy of the distal anterior cerebral artery. J.Neurosurg. 1978; 49: 204-28.

Snickers FD and Drake CG. Aneurysms of the distal anterior cerebral artery. A report of 24 verified cases. South African Med. 1973; 47: 1787-91.
Stefani MA, Schneider FL, Marrone AC, Severino AG, Jackowski AP and Wallace MC. Anatomic variations of anterior cerebral artery cortical branches. Clin.Anat. 2000; 13(4): 231-36.

Umansky F, Dujovny M, Ausman JI, Diaz FG and Mirchandani HG. Anomalies and Variations of the Middle cerebral artery:a microanatomical study. Neurosurgery. 1988; 22(6): 1023-27.

Umansky F, Juarez SM, Dujovny M, Ausman JI, Diaz FG, Gomes F, et al. Microsurgical anatomy of the proximal segments of the middle cerebral artery. J.Neurosurgery. 1984; 61(3): 458-67.

Zurada A and Gielecki JS. A novel formula for the classification of blood vessels according to symmetry, asymmetry and hypoplasia. Folia Morphol.(Warsz.). 2007; 66(4): 339-45.

Received: October 01, 2011;

Revised: March 22, 2012; Accepted: October 18, 2012. 